ELECTRONIC RESEARCH ANNOUNCEMENTS OF THE AMERICAN MATHEMATICAL SOCIETY

Volume 10, Pages 1-10 (February 18, 2004)

S $1079-6762(04) 00124-6$

\title{
COMPLETING LIE ALGEBRA ACTIONS TO LIE GROUP ACTIONS
}

\author{
FRANZ W. KAMBER AND PETER W. MICHOR
}

(Communicated by Alexandre Kirillov)

\begin{abstract}
For a finite-dimensional Lie algebra $\mathfrak{g}$ of vector fields on a manifold $M$ we show that $M$ can be completed to a $G$-space in a universal way, which however is neither Hausdorff nor $T_{1}$ in general. Here $G$ is a connected Lie group with Lie-algebra $\mathfrak{g}$. For a transitive $\mathfrak{g}$-action the completion is of the form $G / H$ for a Lie subgroup $H$ which need not be closed. In general the completion can be constructed by completing each $\mathfrak{g}$-orbit.
\end{abstract}

1. Introduction. In [7, Palais investigated when one could extend a local Lie group action to a global one. He did this in the realm of non-Hausdorff manifolds, since he showed that completing a vector field $X$ on a Hausdorff manifold $M$ may already lead to a non-Hausdorff manifold on which the additive group $\mathbb{R}$ acts. We reproved this result in 3], being unaware of Palais' result. In 4 this result was extended to infinite dimensions and applied to partial differential equations like Burgers' equation: solutions of the PDE were continued beyond the shocks and the universal completion was identified.

Here we give a detailed description of the universal completion of a Hausdorff $\mathfrak{g}$-manifold to a $G$-manifold. For a homogeneous $\mathfrak{g}$-manifold (where the finitedimensional Lie algebra $\mathfrak{g}$ acts infinitesimally transitive) we show that the $G$ completion (for a Lie group $G$ with Lie algebra $\mathfrak{g}$ ) is a homogeneous space $G / H$ for a possibly non-closed Lie subgroup $H$ (Theorem 7). In Example 8 we show that each such situation can indeed be realized. For general $\mathfrak{g}$-manifolds we show that one can complete each $\mathfrak{g}$-orbit separately and replace the $\mathfrak{g}$-orbits in $M$ by the resulting $G$-orbits to obtain the universal completion ${ }_{G} M$ (Theorem 9). All $\mathfrak{g}$ invariant structures on $M$ 'extend' to $G$-invariant structures on ${ }_{G} M$. The relation between our results and those of Palais are described in Section 10.

2. $\mathfrak{g}$-manifolds. Let $\mathfrak{g}$ be a Lie algebra. A $\mathfrak{g}$-manifold is a (finite-dimensional Hausdorff) connected manifold $M$ together with a homomorphism of Lie algebras $\zeta=\zeta^{M}: \mathfrak{g} \rightarrow \mathfrak{X}(M)$ into the Lie algebra of vector fields on $M$. We may assume without loss of generality that it is injective; if not, replace $\mathfrak{g}$ by $\mathfrak{g} / \operatorname{ker}(\zeta)$. We shall also say that $\mathfrak{g}$ acts on $M$.

Received by the editors October 27, 2003.

2000 Mathematics Subject Classification. Primary 22F05, 37C10, 54H15, 57R30, 57S05.

Key words and phrases. $\mathfrak{g}$-manifold, $G$-manifold, foliation.

FWK and PWM were supported by 'Fonds zur Förderung der wissenschaftlichen Forschung, Projekt P 14195 MAT'. 
The image of $\zeta$ spans an integrable distribution on $M$, which need not be of constant rank. So through each point of $M$ there is a unique maximal leaf of that distribution; we also call it the $\mathfrak{g}$-orbit through that point. It is an initial submanifold of $M$ in the sense that a mapping from a manifold into the orbit is smooth if and only if it is smooth into $M$; see [5, 2.14ff.].

Let $\ell: G \times M \rightarrow M$ be a left action of a Lie group with Lie algebra $\mathfrak{g}$. Let $\ell_{a}: M \rightarrow M$ and $\ell^{x}: G \rightarrow M$ be given by $\ell_{a}(x)=\ell^{x}(a)=\ell(a, x)=a . x$ for $a \in G$ and $x \in M$. For $X \in \mathfrak{g}$ the fundamental vector field $\zeta_{X}=\zeta_{X}^{M} \in \mathfrak{X}(M)$ is given by $\zeta_{X}(x)=-T_{e}\left(\ell^{x}\right) \cdot X=-T_{(e, x)} \ell \cdot\left(X, 0_{x}\right)=-\left.\partial_{t}\right|_{0} \exp (t X) \cdot x$. The minus sign is necessary so that $\zeta: \mathfrak{g} \rightarrow \mathfrak{X}(M)$ becomes a Lie algebra homomorphism. For a right action the fundamental vector field mapping without minus would be a Lie algebra homomorphism. Since left actions are more common, we stick to them.

3. The graph of the pseudogroup. Let $M$ be a $\mathfrak{g}$-manifold, effective and connected, so that the action $\zeta=\zeta^{M}: \mathfrak{g} \rightarrow \mathfrak{X}(M)$ is injective. Recall from [1, 2.3] that the pseudogroup $\Gamma(\mathfrak{g})$ consists of all diffeomorphisms of the form

$$
\mathrm{Fl}_{t_{n}}^{\zeta X_{n}} \circ \ldots \circ \mathrm{Fl}_{t_{2}}^{\zeta_{X_{2}}} \circ \mathrm{Fl}_{t_{1}}^{\zeta_{X_{1}}} \mid U
$$

where $X_{i} \in \mathfrak{g}, t_{i} \in \mathbb{R}$, and $U \subset M$ are such that $\mathrm{Fl}_{t_{1}}^{\zeta X_{1}}$ is defined on $U, \mathrm{Fl}_{t_{2}}^{\zeta X_{2}}$ is defined on $\mathrm{Fl}_{t_{1}}^{\zeta_{X_{1}}}(U)$, and so on.

Now we choose a connected Lie group $G$ with Lie algebra $\mathfrak{g}$, and we consider the integrable distribution of constant $\operatorname{rank} d=\operatorname{dim}(\mathfrak{g})$ on $G \times M$ which is given by

$$
\left\{\left(L_{X}(g), \zeta_{X}^{M}(x)\right):(g, x) \in G \times M, X \in \mathfrak{g}\right\} \subset T G \times T M,
$$

where $L_{X}$ is the left invariant vector field on $G$ generated by $X \in \mathfrak{g}$. This gives rise to the foliation $\mathcal{F}_{\zeta}$ on $G \times M$, which we call the graph foliation of the $\mathfrak{g}$-manifold $M$.

Consider the following diagram, where $L(e, x)$ is the leaf through $(e, x)$ in $G \times M$, $\mathcal{O}_{\mathfrak{g}}(x)$ is the $\mathfrak{g}$-orbit through $x$ in $M$, and $W_{x} \subset G$ is the image of the leaf $L(e, x)$ in $G$. Note that $\operatorname{pr}_{1}: L(e, x) \rightarrow W_{x}$ is a local diffeomorphism for the smooth structure of $L(e, x)$.

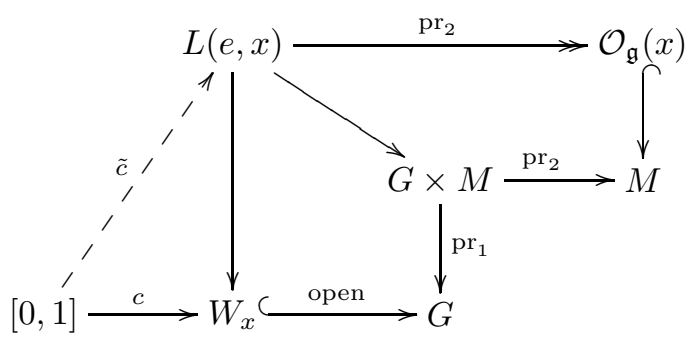

Moreover we consider a piecewise smooth curve $c:[0,1] \rightarrow W_{x}$ with $c(0)=e$ and we assume that it is liftable to a smooth curve $\tilde{c}:[0,1] \rightarrow L(e, x)$ with $\tilde{c}(0)=(e, x)$. Its endpoint $\tilde{c}(1) \in L(e, x)$ does not depend on small (i.e. liftable to $L(e, x)$ ) homotopies of $c$ which respect the ends. This lifting depends smoothly on the choice of the initial point $x$ and gives rise to a local diffeomorphism $\gamma_{x}(c): U \rightarrow\{e\} \times U \rightarrow$ $\{c(1)\} \times U^{\prime} \rightarrow U^{\prime}$, a typical element of the pseudogroup $\Gamma(\mathfrak{g})$ which is defined near $x$. See [1, 2.3] for more information and Example 4 below. Note that the leaf $L(g, x)$ through $(g, x)$ is given by

$$
L(g, x)=\{(g h, y):(h, y) \in L(e, x)\}=\left(\mu_{g} \times \operatorname{Id}\right)(L(e, x)),
$$


where $\mu: G \times G \rightarrow G$ is the multiplication and $\mu_{g}(h)=g h=\mu^{h}(g)$.

4. Examples. It is helpful to keep the following examples in mind, which elaborate upon [1, 5.3]. Let $G=\mathfrak{g}=\mathbb{R}^{2}$, let $W$ be an annulus in $\mathbb{R}^{2}$ containing 0 , and let $M_{1}$ be a simply connected piece of finite or infinite length of the universal cover of $W$. Then the Lie algebra $\mathfrak{g}=\mathbb{R}^{2}$ acts on $M$ but not the group. Let $p: M_{1} \rightarrow W$ be the restriction of the covering map, a local diffeomorphism.

Here $G \times{ }_{\mathfrak{g}} M_{1} \cong G=\mathbb{R}^{2}$. Namely, the graph distribution is then also transversal to the fiber of $\operatorname{pr}_{2}: G \times M_{1} \rightarrow M_{1}$ (since the action is transitive and free on $M_{1}$ ), thus describes a principal $G$-connection on the bundle $\operatorname{pr}_{2}: G \times M_{1} \rightarrow M_{1}$. Each leaf is a covering of $M_{1}$ and hence diffeomorphic to $M_{1}$ since $M_{1}$ is simply connected. For $g \in \mathbb{R}^{2}$ consider $j_{g}: M_{1} \stackrel{\text { ins }_{g}}{\longrightarrow}\{g\} \times M_{1} \subset G \times M_{1} \stackrel{\pi}{\longrightarrow} G \times{ }_{\mathfrak{g}} M_{1}$ and two points $x \neq y \in M_{1}$. We may choose a smooth curve $\gamma$ in $M_{1}$ from $x$ to $y$, lift it into the leaf $L(g, x)$ and project it to a curve $c$ in $g+W$ from $g$ to $c(1)=g+p(y)-p(x) \in g+W$. Then $(g, x)$ and $(c(1), y)$ are on the same leaf. So $j_{g}(x)=j_{g}(y)$ if and only if $p(x)=p(y)$. So we see that $j_{g}(x)=g+p(x)$, and thus $G \times{ }_{\mathfrak{g}} M_{1}=\mathbb{R}^{2}$. This will also follow from 7 .

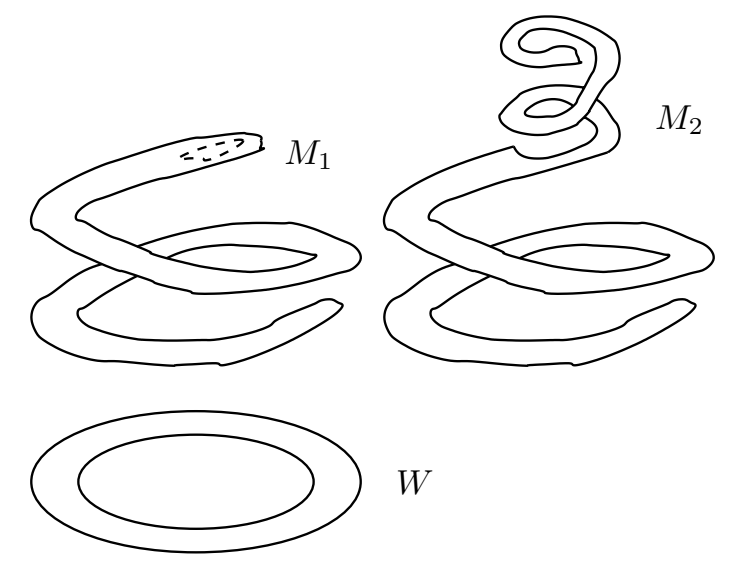

Let us further complicate the situation by now omitting a small disk in $M_{1}$ so that it becomes non-simply connected but still projects onto $W$, and let $M_{2}$ be a simply connected component of the universal cover of $M_{1}$ with the disk omitted. What happens now is that homotopic curves which act equally on $M_{1}$ act differently on $M_{2}$.

It is easy to see with the methods described below that the completion ${ }_{G} M_{i}=\mathbb{R}^{2}$ in both cases.

5. Enlarging to group actions. In the situation of Section 3 let us denote by ${ }_{G} M=G \times{ }_{\mathfrak{g}} M=G \times M / \mathcal{F}_{\zeta}$ the space of leaves of the foliation $\mathcal{F}_{\zeta}$ on $G \times M$, with the quotient topology. For each $g \in G$ we consider the mapping

$$
j_{g}: M \stackrel{\text { ins }_{g}}{\longrightarrow}\{g\} \times M \subset G \times M \stackrel{\pi}{\longrightarrow} M=G \times \times_{\mathfrak{g}} M .
$$

Note that the submanifolds $\{g\} \times M \subset G \times M$ are transversal to the graph foliation $\mathcal{F}_{\zeta}$. The leaf space ${ }_{G} M$ of $G \times M$ admits a unique smooth structure, possibly singular and non-Hausdorff, such that a mapping $f:{ }_{G} M \rightarrow N$ into a smooth manifold $N$ is smooth if and only if the compositions $f \circ j_{g}: M \rightarrow N$ are smooth. For example 
we may use the structure of a Frölicher space or smooth space induced by the mappings $j_{g}$ in the sense of [6] Section 23] on ${ }_{G} M=G \times{ }_{\mathfrak{g}} M$. The canonical open maps $j_{g}: M \rightarrow{ }_{G} M$ for $g \in G$ are called the charts of ${ }_{G} M$. By construction, for each $x \in M$ and for $g^{\prime} g^{-1}$ near enough to $e$ in $G$ there exists a curve $c:[0,1] \rightarrow W_{x}$ with $c(0)=e$ and $c(1)=g^{\prime} g^{-1}$ and an open neighborhood $U$ of $x$ in $M$ such that for the smooth transformation $\gamma_{x}(c)$ in the pseudogroup $\Gamma(\mathfrak{g})$ we have

$$
j_{g^{\prime}} \mid U=j_{g} \circ \gamma_{x}(c) \text {. }
$$

Thus the mappings $j_{g}$ may serve as a replacement for charts in the description of the smooth structure on ${ }_{G} M$. Note that the mappings $j_{g}$ are not injective in general. Even if $g=g^{\prime}$, there might be liftable smooth loops $c$ in $W_{x}$ such that (5.2) holds. Note also some similarity of the system of 'charts' $j_{g}$ with the notion of an orbifold where one uses finite groups instead of pseudogroup transformations.

The leaf space ${ }_{G} M=G \times \times_{\mathfrak{g}} M$ is a smooth $G$-space where the $G$-action is induced by $\left(g^{\prime}, x\right) \mapsto\left(g g^{\prime}, x\right)$ in $G \times M$.

Theorem. The $G$-completion ${ }_{G} M$ has the following universal properties:

(5.3) Given any Hausdorff $G$-manifold $N$ and $\mathfrak{g}$-equivariant mapping $f: M \rightarrow N$ there exists a unique $G$-equivariant continuous mapping $\tilde{f}:{ }_{G} M \rightarrow N$ with $\tilde{f} \circ j_{e}=f$. Namely, the mapping $\bar{f}: G \times M \rightarrow N$ given by $\bar{f}(g, x)=g . f(x)$ is smooth and factors to $\tilde{f}:{ }_{G} M \rightarrow N$.

(5.4) In the setting of (5.3), the universal property holds also for the $T_{1}$-quotient of ${ }_{G} M$, which is given as the quotient $G \times M / \overline{\mathcal{F}}_{\zeta}$ of $G \times M$ by the equivalence relation generated by the closure of leaves.

(5.5) If $M$ carries a symplectic or Poisson structure or a Riemannian metric such that the $\mathfrak{g}$-action preserves this structure or is even a Hamiltonian action, then the structure 'can be extended to ${ }_{G} M$ so that the enlarged $G$-action preserves these structures or is even Hamiltonian'.

Proof. (5.3) Consider the mapping $\bar{f}=\ell^{N} \circ\left(\operatorname{Id}_{G} \times f\right): G \times M \rightarrow N$ which is given by $\bar{f}(g, x)=g . f(x)$. Then by (3.1) and (3.2) we have for $X \in G$

$$
\begin{aligned}
T \bar{f} \cdot\left(L_{X}(g), \zeta_{X}^{M}(x)\right) & =T \ell \cdot\left(L_{X}(g), T_{x} f \cdot \zeta_{X}^{M}(x)\right) \\
& =T \ell \cdot\left(R_{\operatorname{Ad}(g) X}(g), 0_{f(x)}\right)+T \ell\left(0_{g}, \zeta_{X}^{N}(f(x))\right) \\
& =-\zeta_{\operatorname{Ad}(g) X}(g \cdot f(x))+T \ell_{g} \cdot \zeta_{X}^{N}(f(x))=0 .
\end{aligned}
$$

Thus $\bar{f}$ is constant on the leaves of the graph foliation on $G \times M$ and thus factors to $\tilde{f}:{ }_{G} M \rightarrow N$. Since $\bar{f}\left(g \cdot g_{1}, x\right)=g \cdot g_{1} \cdot f(x)=g \cdot \bar{f}(g, x)$, the mapping $\tilde{f}$ is $G$ equivariant. Since $N$ is Hausdorff, $\tilde{f}$ is even constant on the closure of each leaf, thus (5.4) holds also.

(5.5) Let us treat Poisson structure $P$ on $M$. For symplectic structures or Riemannian metrics the argument is similar and simpler. Since the Lie derivative along fundamental vector fields of $P$ vanishes, the pseudogroup transformation $\gamma_{x}(c)$ in (5.2) preserves $P$. Since ${ }_{G} M$ is the quotient of the disjoint union of all spaces $\{g\} \times M$ for $g \in G$ under the equivalence relation described by (5.2), $P$ 'passes down to this quotient'. Note that we refrain from putting too much meaning on this statement.

The universal property (5.3) holds also for smooth $G$-spaces $N$ which need not be Hausdorff, nor $T_{1}$, but should have tangent spaces and foliations so that it is 
meaningful to talk about $\mathfrak{g}$-equivariant mappings. We will not go into this, but see [6, Section 23], for some concepts which point in this direction.

As an application of the universal property of the $G$-completion ${ }_{G} M$, we see that ${ }_{G} M$ depends on the choice of $G$ in the following way. We write $G=\Gamma \backslash \widetilde{G}$, where $\widetilde{G}$ is the simply connected Lie group with Lie algebra $\mathfrak{g}$ and $\Gamma \subset \widetilde{G}$ is the discrete central subgroup such that $\Gamma \cong \pi_{1}(G)$. Then we have ${ }_{G} M \cong \Gamma \backslash_{\tilde{G}} M$ as $G$-spaces, so that $\widetilde{G}^{M}$ is potentially less singular than ${ }_{G} M$.

6. Example. Let $\mathfrak{g}=\mathbb{R}^{2}$ with basis $X, Y$, let $M=\mathbb{R}^{3} \backslash\{(0,0, z): z \in \mathbb{R}\}$, and let $\zeta^{\alpha}: \mathfrak{g} \rightarrow \mathfrak{X}(M)$ be given by

$$
\zeta_{X}^{\alpha}=\partial_{x}+\alpha \frac{y z}{x^{2}+y^{2}} \partial_{z}, \quad \zeta_{Y}^{\alpha}=\partial_{y}-\alpha \frac{x z}{x^{2}+y^{2}} \partial_{z}, \quad \alpha>0
$$

which satisfy $\left[\zeta_{X}^{\alpha}, \zeta_{Y}^{\alpha}\right]=0$. By construction of the graph foliation $\mathcal{F}_{\zeta^{\alpha}}$ in (3.1) and the procedure summarized in diagram (3.2), the leaves of $\mathcal{F}_{\zeta^{\alpha}}$ are determined explicitly as follows. For any smooth curve $c(t)=(\xi(t), \eta(t)) \in G$ starting at $\left(\xi_{0}, \eta_{0}\right)$ we have $\dot{c}(t)=\dot{\xi}(t) X+\dot{\eta}(t) Y \in \mathfrak{g}$ and the lifted curve $(c(t), \mathbf{y}(t))$ is in the leaf $L\left(\left(\xi_{0}, \eta_{0}\right), \mathbf{y}_{0}\right)$ if and only if it satisfies the first order ODE

$$
(\mathbf{y}(t), \dot{\mathbf{y}}(t))=\dot{\xi}(t) \zeta_{X}^{\alpha}(\mathbf{y}(t))+\dot{\eta}(t) \zeta_{Y}^{\alpha}(\mathbf{y}(t))
$$

with initial value $\mathbf{y}(0)=\mathbf{y}_{0}=\left(x_{0}, y_{0}, u=z_{0}\right) \in M$. Substituting (6.1) into (6.2), we see that this ODE is linear, that is, $\dot{x}=\dot{\xi}, \dot{y}=\dot{\eta}$ and $\dot{z}=-\alpha z \frac{x \dot{\eta}-y \dot{\xi}}{r^{2}}=-\alpha z \frac{x \dot{y}-y \dot{x}}{r^{2}}$, where $r^{2}=x^{2}+y^{2}$. Thus the projection $\mathbf{x}(t)$ of $\mathbf{y}(t)$ to the $(x, y)$-plane is given by $\mathbf{x}(t)=c(t)-\left(\left(\xi_{0}, \eta_{0}\right)-\mathbf{x}_{0}\right)=c(t)-\left(\xi_{0}-x_{0}, \eta_{0}-y_{0}\right)$, whereas the third equation leads to

$$
z(t)=u e^{-\alpha \int_{0}^{t} d \theta}=u e^{-\alpha\left(\theta(t)-\theta_{0}\right)}=u e^{\alpha \theta_{0}} e^{-\alpha \theta(t)},
$$

where $\theta$ is the angle function in the $(x, y)$-plane. This depends only on the endpoints $\mathbf{x}_{0}, \mathbf{x}(t)$ and the winding number of the curve $\mathbf{x}$ and is otherwise independent of $\mathbf{x}$. Incompleteness occurs whenever the curve $\mathbf{x}$ goes to $(0,0) \in \mathbb{R}^{2}$ in finite time $\bar{t}<\infty$, that is, $\mathbf{x}(t) \rightarrow(0,0), t \uparrow \bar{t}$, or equivalently $c(t) \rightarrow\left(\xi_{0}, \eta_{0}\right)-\mathbf{x}_{0}, t \uparrow \bar{t}$. It follows that the leaf $L\left(\left(\xi_{0}, \eta_{0}\right), \mathbf{y}_{0}\right)$ is parametrized by $(r, \theta) \in \mathbb{R}_{+} \times \mathbb{R}$ with $z=z(\theta)$ being independent of $r>0$ and that

$$
\operatorname{pr}_{1}: L\left(\left(\xi_{0}, \eta_{0}\right), \mathbf{y}_{0}\right) \rightarrow W_{\left(\xi_{0}, \eta_{0}\right), \mathbf{y}_{0}}=\mathbb{R}^{2} \backslash\left\{\left(\xi_{0}, \eta_{0}\right)-\mathbf{x}_{0}\right\}
$$

in (3.2) is a universal covering. This is visibly consistent with (3.3). In order to parametrize the space of leaves ${ }_{G} M$, we observe that the parameter $\mathbf{x}_{0}$ can be eliminated. In fact, from the previous formulas we see that

$$
L\left(\left(\xi_{0}^{\prime}, \eta_{0}^{\prime}\right),\left(\mathbf{x}_{0}^{\prime}, u^{\prime}\right)\right)=L\left(\left(\xi_{0}, \eta_{0}\right),\left(\mathbf{x}_{0}, u\right)\right)
$$

if and only if $\left(\xi_{0}^{\prime}, \eta_{0}^{\prime}\right)-\mathbf{x}_{0}^{\prime}=\left(\xi_{0}, \eta_{0}\right)-\mathbf{x}_{0}$ and $u^{\prime}=u e^{\alpha\left(\theta_{0}-\theta_{0}^{\prime}\right)}$, so that we have $z^{\prime}(\theta)=u^{\prime} e^{\alpha \theta_{0}^{\prime}} e^{-\alpha \theta(t)}=u e^{\alpha \theta_{0}} e^{-\alpha \theta(t)}=z(\theta)$. In particular, it follows that

$$
L\left(\left(\xi_{0}, \eta_{0}\right), \mathbf{y}_{0}\right)=L\left(\left(\xi_{0}^{\prime}+1, \eta_{0}^{\prime}\right),\left(1,0, u^{\prime}\right)\right),
$$

where $\left(\xi_{0}^{\prime}, \eta_{0}^{\prime}\right)=\left(\xi_{0}, \eta_{0}\right)-\mathbf{x}_{0}, u^{\prime}=u e^{\alpha \theta_{0}}, \theta_{0}^{\prime}=0$, projecting to $\mathbb{R}^{2} \backslash\left\{\left(\xi_{0}^{\prime}, \eta_{0}^{\prime}\right)\right\}$. Therefore the leaves of the form $L\left(\left(\xi_{0}+1, \eta_{0}\right),(1,0, u)\right)$ are distinct for different values of $\left(\xi_{0}, \eta_{0}\right)$ and fixed value of $u$ and from the relation (3.3) we conclude that

$$
L\left(\left(\xi_{0}+1, \eta_{0}\right),(1,0, u)\right)=\left(\xi_{0}, \eta_{0}\right)+L((1,0),(1,0, u)),
$$

that is, $G=\mathbb{R}^{2}$ acts without isotropy on ${ }_{G} M$. We also need to determine the range for the parameter $u$. Obviously, we have $L\left((1,0),\left(1,0, u^{\prime}\right)\right)=L((1,0),(1,0, u))$ 
if and only if $u^{\prime}=e^{2 \pi \alpha n} u$ for $n \in \mathbb{Z}$. Thus these leaves are parametrized by $[u]$, taking values in the quotient of the additive group $\mathbb{R}$ under the multiplicative group $\left\{e^{2 \pi \alpha n}: n \in \mathbb{Z}\right\}$, that is,

$$
\{0\} \cup \mathbb{S}_{+}^{1} \cup \mathbb{S}_{-}^{1} \cong\{0\} \cup \mathbb{R}_{+}^{\times} /\left\{e^{2 \pi \alpha n}: n \in \mathbb{Z}\right\} \cup \mathbb{R}_{-}^{\times} /\left\{e^{2 \pi \alpha n}: n \in \mathbb{Z}\right\} .
$$

The topology on the above space is determined by the leaf closures, respectively the orbit closures. First we have $\overline{L\left(\left(\xi_{0}+1, \eta_{0}\right),(1,0, u)\right)}=\left(\xi_{0}, \eta_{0}\right)+\overline{L((1,0),(1,0, u))}$ in $G \times M$, and it is sufficient to determine the closures of $L((1,0),(1,0, u))$. For $(1,0, u) \in M$ with $u \neq 0$ we consider the curve $c(\theta)=e^{i \theta} \in G=\mathbb{R}^{2}$. It is liftable to $G \times M$ and determines on $M$ the curve $\mathbf{y}(t)=\left(\cos \theta, \sin \theta, u e^{-\alpha \theta}\right)$. Thus the curve $(c(\theta), \mathbf{y}(\theta))$ in the leaf through $(1,0 ; 1,0, u) \in G \times M \subset \mathbb{R}^{5}$ has a limit cycle for $\theta \rightarrow \infty$ which lies in the different leaf through $(1,0 ; 1,0,0)$, which is closed, given by the $(x, y)$-plane $\left(\mathbb{R}^{2} \times 0\right) \backslash 0$ at level $(1,0) \in G$. Thus we have

$$
\overline{L((1,0),(1,0, u))}=L((1,0),(1,0, u)) \cup L((1,0),(1,0,0)) .
$$

Hence the leaf $L((1,0),(1,0, u))$ is not closed, and the topological space ${ }_{G} M$ is not $T_{1}$ and not a manifold. The orbits of the $\mathfrak{g}$-action are determined by the leaf structure via $\mathrm{pr}_{2}$ in diagram (3.2), and they look here as follows. The $(x, y)$-plane $\left(\mathbb{R}^{2} \times 0\right) \backslash 0$ is a closed orbit. Orbits above this plane are helicoidal staircases leading down and accumulating exponentially at the $(x, y)$-plane. Orbits below this plane are helicoidal staircases leading up and again accumulating exponentially. Thus the orbit space $M / \mathfrak{g}$ of the $\mathfrak{g}$-action is given by (6.8), with the point 0 being closed. By (6.9), the closure of any orbit represented by a point $[u]$ on one of the circles is given by $\{[u], 0\}$. From (6.6) and (6.7), we see that the $G$-completion ${ }_{G} M$ has a section over the orbit space ${ }_{G} M / G \cong M / \mathfrak{g}$ given by $[u] \mapsto L((1,0),(1,0, u))$. Therefore ${ }_{G} M \cong G \times M / \mathfrak{g}=\mathbb{R}^{2} \times\left\{\{0\} \cup \mathbb{S}_{+}^{1} \cup \mathbb{S}_{-}^{1}\right\}$.

The structure of the completion and the orbit spaces are independent of the deformation parameter $\alpha>0$ in (6.1). However, for $\alpha \downarrow 0$, the completion just means adding in the $z$-axis, that is, we get ${ }_{G} M \cong \mathbb{R}^{3}$ with $G=\mathbb{R}^{2}$ acting by parallel translation on the affine planes $z=c$, and $M / \mathfrak{g} \cong{ }_{G} M / G \cong \mathbb{R}$ as it should be.

It was pointed out to us 2 that one can make this example still more pathological. Consider the above example only in a cylinder over the annulus $0<x^{2}+y^{2}<1$. Add an open handle to the disk and continue the $\mathbb{R}^{2}$-action on the cylinder over the disk with an open handle added in such a way that there is a shift in the $z$-direction when one traverses the handle. Then one of the helicoidal staircases is connected to the the disk itself, so it accumulates onto itself. This is called a 'resilient leaf' in foliation theory.

7. Theorem. Let $M$ be a connected transitive effective $\mathfrak{g}$-manifold. Let $G$ be a connected Lie group with Lie algebra $\mathfrak{g}$. Then we have:

(7.1) There exists a subgroup $H \subset G$ such that the $G$-completion ${ }_{G} M$ is diffeomorphic to $G / H$.

(7.2) The Hausdorff quotient of ${ }_{G} M$ is the homogeneous manifold $G / \bar{H}$. It has the following universal property: For each smooth $\mathfrak{g}$-equivariant mapping $f: M \rightarrow N$ into a Hausdorff $G$-manifold $N$ there exists a unique smooth $G$-equivariant mapping $\tilde{f}: G / \bar{H} \rightarrow N$ with $f=\tilde{f} \circ \pi \circ j_{e}: M \rightarrow G / H \stackrel{\pi}{\longrightarrow}$ $G / \bar{H} \rightarrow N$. 
(7.3) For each leaf $L\left(g, x_{0}\right) \subset G \times M$ the projection $\operatorname{pr}_{2}: L\left(g, x_{0}\right) \rightarrow M$ is a smooth fiber bundle with typical fiber $H$.

Proof. (7.1) We choose a base point $x_{0} \in M$. The $G$-completion is given by ${ }_{G} M=$ $G \times \mathfrak{g} M$, the orbit space of the $\mathfrak{g}$-action on $G \times M$ which is given by $\mathfrak{g} \ni X \mapsto$ $L_{X} \times \zeta_{X}^{M}$, and the $G$-action on the completion is given by multiplication from the left. The submanifold $G \times\left\{x_{0}\right\}$ meets each $\mathfrak{g}$-orbit in $G \times M$ transversely, since

$$
\begin{aligned}
T_{\left(g, x_{0}\right)}\left(G \times\left\{x_{0}\right\}\right)+T_{\left(g, x_{0}\right)} L\left(g, x_{0}\right) & =\left\{L_{X}(g) \times 0_{x_{0}}+L_{Y}(g) \times \zeta_{Y}\left(x_{0}\right): X, Y \in \mathfrak{g}\right\} \\
& =T_{\left(g, x_{0}\right)}(G \times M) .
\end{aligned}
$$

By (3.3) we have $L(g, x)=g \cdot L(e, x)$ so that the isotropy Lie algebra $\mathfrak{h}=\mathfrak{g}_{x_{0}}=$ $\left\{X \in \mathfrak{g}: \zeta_{X}\left(x_{0}\right)=0\right\}$ is also given by

$$
\begin{aligned}
X \in \mathfrak{h} & \Longleftrightarrow X \times 0_{x_{0}} \in T_{\left(e, x_{0}\right)}\left(G \times\left\{x_{0}\right\}\right) \cap T_{\left(e, x_{0}\right)} L\left(e, x_{0}\right) \\
& \Longleftrightarrow L_{X}(g) \times 0_{x_{0}} \in T_{\left(g, x_{0}\right)}\left(G \times\left\{x_{0}\right\}\right) \cap T_{\left(g, x_{0}\right)} L\left(g, x_{0}\right) .
\end{aligned}
$$

Since $G \times\left\{x_{0}\right\}$ is a leaf of a foliation and the $L(e, x)$ also form a foliation, $\mathfrak{h}$ is a Lie subalgebra of $\mathfrak{g}$. Let $H_{0}$ be the connected Lie subgroup of $G$ which corresponds to $\mathfrak{h}$. Then clearly $H_{0} \times\left\{x_{0}\right\} \subset G \times\left\{x_{0}\right\} \cap L\left(e, x_{0}\right)$. Let the subgroup $H \subset G$ be given by

$$
H=\left\{g \in G:\left(g, x_{0}\right) \in L\left(e, x_{0}\right)\right\}=\left\{g \in G: L\left(g, x_{0}\right)=L\left(e, x_{0}\right)\right\}
$$

then the $C^{\infty}$-curve component of $H$ containing $e$ is just $H_{0}$. So $H$ consists of at most countably many $H_{0}$-cosets. Thus $H$ is a Lie subgroup of $G$ (with a finer topology, perhaps). By construction the orbit space $G \times \mathfrak{g} M$ equals the quotient of the transversal $G \times\left\{x_{0}\right\}$ by the relation induced by intersecting with each leaf $L\left(g, x_{0}\right)$ separately, i.e., $G \times_{\mathfrak{g}} M=G / H$.

(7.2) Obviously the $T_{1}$-quotient of $G / H$ equals the Hausdorff quotient $G / \bar{H}$, which is a smooth manifold. The universal property is easily seen.

(7.3) Let $x \in M$ and $(g, x) \in L\left(e, x_{0}\right)=L(g, x)=g . L(e, x)$. So it suffices to treat the leaf $L(e, x)$. We choose $X_{1}, \ldots, X_{n} \in \mathfrak{g}$ such that $\zeta_{X_{1}}(x), \ldots, \zeta_{X_{n}}(x)$ form a basis of the tangent space $T_{x} M$. Let $u: U \rightarrow \mathbb{R}^{n}$ be a chart on $M$ centered at $x$ such that $u(U)$ is an open ball in $\mathbb{R}^{n}$ and such that $\zeta_{X_{1}}(y), \ldots, \zeta_{X_{n}}(y)$ are still linearly independent for all $y \in U$. For $y \in U$ consider the smooth curve $c_{y}:[0,1] \rightarrow U$ given by $c_{y}(t)=u^{-1}(t . u(y))$. We consider

$$
\begin{aligned}
\partial_{t} c_{y}(t) & =c_{y}^{\prime}(t)=\sum_{i=1}^{n} f_{y}^{i}(t) \zeta_{X_{i}}\left(c_{y}(t)\right), \quad f_{y}^{i} \in C^{\infty}([0,1], \mathbb{R}), \\
X_{y}(t) & =\sum_{i=1}^{n} f_{y}^{i}(t) X_{i} \in \mathfrak{g}, \quad X \in C^{\infty}([0,1], \mathfrak{g}), \\
g_{y} & \in C^{\infty}([0,1], G), \quad T\left(\mu_{g_{y}(t)}\right) \partial_{t} g_{y}(t)=X_{y}(t), \quad g_{y}(0)=e,
\end{aligned}
$$

and everything is also smooth in $y \in U$. Then for $h \in H$ we have $\left(h . g_{y}(t), c_{y}(t)\right) \in$ $L(e, x)$ since

$$
\partial_{t}\left(h . g_{y}(t), c_{y}(t)\right)=\left(L_{X_{y}(t)}\left(h \cdot g_{y}(t)\right), \zeta_{X_{y}(t)}\left(c_{y}(t)\right)\right) .
$$

Thus $U \times H \ni(y, h) \mapsto \operatorname{pr}_{2}^{-1}(U) \cap L(e, x)$ is the required fiber bundle parameterization. 
8. Example. Let $G$ be a simply connected Lie group and let $H$ be a connected Lie subgroup of $G$ which is not closed. For example, let $G=\operatorname{Spin}(5)$, which is compact of rank 2, and let $H$ be a dense 1-parameter subgroup in its 2-dimensional maximal torus. Let $\operatorname{Lie}(G)=\mathfrak{g}$ and $\operatorname{Lie}(H)=\mathfrak{h}$. We consider the foliation of $G$ into right $H$-cosets $g H$ which is generated by $\left\{L_{X}: X \in \mathfrak{h}\right\}$ and is left invariant under $G$. Let $U$ be a chart centered at $e$ on $G$ which is adapted to this foliation, i.e. $u: U \rightarrow u(U)=V_{1} \times V_{2} \subset \mathbb{R}^{k} \times \mathbb{R}^{n-k}$ such that the sets $u^{-1}\left(V_{1} \times\{x\}\right)$ are the leaves intersected with $U$. We assume that $V_{1}$ and $V_{2}$ are open balls, and that $U$ is so small that exp : $W \rightarrow U$ is a diffeomorphism for a suitable convex open set $W \subset \mathfrak{g}$. Of course, $\mathfrak{g}$ acts on $U$ and respects the foliation, so this $\mathfrak{g}$-action descends to the leaf space $M$ of the foliation on $U$ which is diffeomorphic to $V_{2}$.

Lemma. In this situation, for the $G$-completion we have $G \times_{\mathfrak{g}} M=G / H$.

Proof. We use the method described in the end of the proof of Theorem 7: ${ }_{G} M=$ $G \times \mathfrak{g} M$ is the quotient of the transversal $G \times\left\{x_{0}\right\}$ by the relation induced by intersecting with each leaf $L\left(g, x_{0}\right)$ separately. Thus we have to determine the subgroup $H_{1}=\left\{g \in G:\left(g, x_{0}\right) \in L(e, g)\right\}$.

Obviously any smooth curve $c_{1}:[0,1] \rightarrow H$ starting at $e$ is liftable to $L\left(e, x_{0}\right)$ since it does not move $x_{0} \in M$. So $H \subseteq H_{1}$, and moreover $H$ is the $C^{\infty}$-path component of the identity in $H_{1}$.

Conversely, if $c=\left(c_{1}, c_{2}\right):[0,1] \rightarrow L\left(e, x_{0}\right) \subset G \times M$ is a smooth curve from $\left(e, x_{0}\right)$ to $\left(g, x_{0}\right)$, then $c_{2}$ is a smooth loop through $x_{0}$ in $M$ and there exists a smooth homotopy $h$ in $M$ which contracts $c_{2}$ to $x_{0}$, fixing the ends. Since $\operatorname{pr}_{2}$ : $L\left(e, x_{0}\right) \rightarrow M$ is a fiber bundle by (7.3), we can lift the homotopy $h$ from $M$ to $L\left(e, x_{0}\right)$ with starting curve $c$, fixing the ends, and deforming $c$ to a curve $c^{\prime}$ in $L\left(e, x_{0}\right) \cap \mathrm{pr}_{2}^{-1}\left(x_{0}\right)$. Then $\operatorname{pr}_{1} \circ c^{\prime}$ is a smooth curve in $H_{1}$ connecting $e$ and $g$.

Thus $H_{1}=H$, and consequently ${ }_{G} M=G / H$.

9. Theorem. Let $M$ be a connected $\mathfrak{g}$-manifold. Let $G$ be a connected Lie group with Lie algebra $\mathfrak{g}$. Then the $G$-completion ${ }_{G} M$ can be described in the following way:

(9.1) Form the leaf space $M / \mathfrak{g}$, a quotient of $M$ which may be non-Hausdorff and not $T_{1}$ etc.

(9.2) For each point $z \in M / \mathfrak{g}$, replace the orbit $\pi^{-1}(z) \subset M$ by the homogeneous space $G / H_{x}$ described in Theorem 7 , where $x$ is some point in the orbit $\pi^{-1}(z) \subset M$. One can use transversals to the $\mathfrak{g}$-orbits in $M$ to describe this in more detail.

(9.3) For each point $z \in M / \mathfrak{g}$, one can also replace the orbit $\pi^{-1}(z) \subset M$ by the homogeneous space $G \overline{H_{x}}$ described in Theorem 7 , where $x$ is some point in the orbit $\pi^{-1}(z) \subset M$. The resulting $G$-space has then Hausdorff orbits which are smooth manifolds, but the same orbit space as $M / \mathfrak{g}$.

See Example 6 above.

Proof. Let $\mathcal{O}(x) \subset M$ be the $\mathfrak{g}$-orbit through $x$, i.e., the leaf through $x$ of the singular foliation (with non-constant leaf dimension) on $M$ which is induced by the $\mathfrak{g}$-action. Then the $G$-completion of the orbit $\mathcal{O}(x)$ is ${ }_{G} \mathcal{O}(x)=G / H_{x}$ for the Lie subgroup $H_{x} \subset G$ described in Theorem (7.1). By the universal property of the $G$-completion we get a $G$-equivariant mapping ${ }_{G} \mathcal{O}(x) \rightarrow{ }_{G} M$ which is injective and a homeomorphism onto its image, since we can repeat the construction of Theorem 
(7.1) on $M$. Clearly the mapping $j_{e}: M \rightarrow{ }_{G} M$ induces a homeomorphism between the orbit spaces $M / \mathfrak{g} \rightarrow{ }_{G} M / G$.

Now let $s: V \rightarrow M$ be an embedding of a submanifold which is a transversal to the $\mathfrak{g}$-foliation at $s\left(v_{0}\right)$. We have $T s \cdot T_{v_{0}} V \oplus \zeta_{s\left(v_{0}\right)}(\mathfrak{g})=T_{s\left(v_{0}\right)} M$. Then $s$ induces a mapping $V \rightarrow G \times M$ and $V \rightarrow{ }_{G} M$ and we may use the point $s(v)$ in replacing $\mathcal{O}(s(v))$ by $G / H_{s(v)}$ for $v$ near $v_{0}$.

The following diagram summarizes the relation between the preceding constructions.

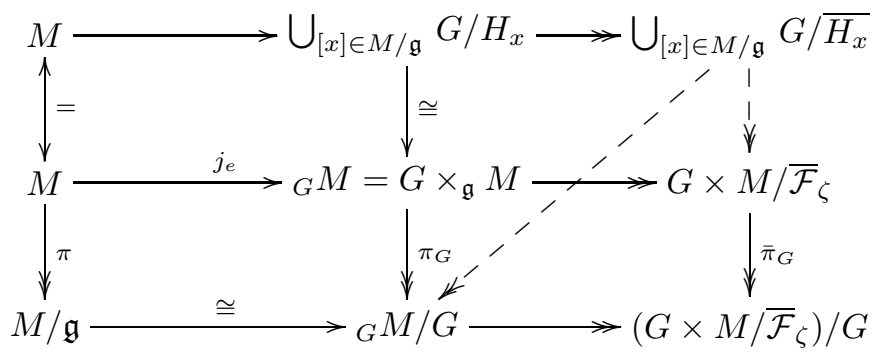

Note that taking the $T_{1}$-quotient $G \times M / \overline{\mathcal{F}}_{\zeta}$ of the leaf space ${ }_{G} M$ may be a very severe reduction. In Example 6 the isotropy groups $H_{x}$ are trivial and we have $G \times M / \overline{\mathcal{F}}_{\zeta}=\mathbb{R}^{2} \times\{0\}$ and $\left(G \times M / \overline{\mathcal{F}}_{\zeta}\right) / G=\{0\}$.

10. Palais' treatment of $\mathfrak{g}$-manifolds. In 7], Palais considered $\mathfrak{g}$-actions on finite-dimensional manifolds $M$ in the following way. He assumed from the beginning that $M$ may be a non-Hausdorff manifold, since the completion may be non-Hausdorff. Then he introduced notions which we can express as follows in the terms introduced here:

(10.1) $(M, \zeta)$ is called generating if it generates a local $G$-transformation group. See [7, II, 2, Def. V and II, 7, Thm. XI]. This holds if and only if the leaves of the graph foliation on $G \times M$ described in Section 3 are Hausdorff. For Hausdorff $\mathfrak{g}$-manifolds this is always the case.

$(10.2)(M, \zeta)$ is called uniform if $\operatorname{pr}_{1}: L(e, x) \rightarrow G$ in (3.2) is a covering map for each $x \in M$. See [7, III, 6, Def. VIII and III, 6, Thm. XVII, Cor., Cor. 2]. In the Hausdorff case the $\mathfrak{g}$-action is then complete and it may be integrated to a $\widetilde{G}$-action, where $\widetilde{G}$ is a simply connected Lie group with Lie algebra $\mathfrak{g}$, so that $\widetilde{G}^{M} \cong M$.

(10.3) $(M, \zeta)$ is called univalent if $\operatorname{pr}_{1}: L(e, x) \rightarrow G$ in (3.2) is injective for all $x$. See [7] III, 2, Def. VI and III, 4, Thm. X].

(10.4) $(M, \zeta)$ is called globalizable if there exists a (non-Hausdorff) $G$-manifold $N$ which contains $M$ equivariantly as an open submanifold. See [7, III, 1, Def. II and III, 4, Thm. X]. This is a severe condition which is not satisfied in examples 4 and 6 above.

Palais' main result on (non-Hausdorff) manifolds with a vector field says that (10.1), (10.3), and (10.4) are equivalent. See [7, III, 7, Thm. XX].

On (non-Hausdorff) $\mathfrak{g}$-manifolds his main result is that (10.3) and (10.4) are equivalent. See [7, III, 1, Def. II and III, 4, Thm. X], and also [7, III, 2, Def. VI and III, 4, Thm. X]. 
11. Concluding remarks. (11.1) A suitable setting for further development might be the class of discrete $\mathfrak{g}$-manifolds, that is, $\mathfrak{g}$-manifolds for which the $\widetilde{G}$ space $\widetilde{G} M$ is $T_{1}$, or equivalently the leaves of the graph foliation $\mathcal{F}_{\zeta}$ on $\widetilde{G} \times M$ are closed. In this case, the charts $j_{g}: M \rightarrow{ }_{G} M$ in (5.1) are local diffeomorphisms with respect to the unique smooth structure on $\widetilde{G}^{M}$, and $\widetilde{G}^{M}$ is a smooth manifold, albeit not necessarily Hausdorff.

(11.2) In the context of (11.1), there are several definitions of proper $\mathfrak{g}$-actions, all of which are equivalent to saying that the $\widetilde{G}$-action on $\widetilde{G}^{M}$ is proper. Many properties of proper actions will carry over to this case.

\section{REFERENCES}

1. D. V. Alekseevsky and Peter W. Michor, Differential geometry of $\mathfrak{g}$-manifolds., Differ. Geom. Appl. 5 (1995), 371-403, arXiv:math.DG/9309214. MR 96k:53035

2. G. Hector, Private communication, 2002.

3. Franz W. Kamber and Peter W. Michor, The flow completion of a manifold with vector field, Electron. Res. Announc. Amer. Math. Soc. 6 (2000), 95-97, math.DG/0007173. MR 2001k:37031

4. Boris Khesin and Peter W. Michor, The flow completion of Burgers' equation, pp. 1-8, Walter de Gruyter, Berlin, 2004, IRMA Lectures in Mathematics and Theoretical Physics. http://www.mat.univie.ac.at/ michor/burgers.ps

5. Ivan Kolář, Jan Slovák, and Peter W. Michor, Natural operators in differential geometry, Springer-Verlag, Heidelberg, Berlin, New York, 1993. MR 94a:58004

6. Andreas Kriegl and Peter W. Michor, The Convenient Setting for Global Analysis, Math. Surveys and Monographs, vol. 53, AMS, Providence, 1997, www.ams.org/online_bks/surv53/. MR 98i:58015

7. Richard S. Palais, A global formulation of the Lie theory of transformation groups, Mem. AMS 22 (1957). MR 22:12162

Department of Mathematics, University of Illinois, 1409 West Green Street, UrBANA, IL 61801

E-mail address: kamber@math.uiuc.edu

Institut für Mathematik, Universität Wien, Nordbergstrasse 15, A-1090 Wien, Austria, and Erwin Schrödinger Institut für Mathematische Physik, Boltzmanngasse 9, A-1090 Wien, Austria

E-mail address: michor@esi.ac.at 